\title{
Study of the Full-Service and Low-Cost Carriers Network Configuration
}

\author{
Oriol Lordan \\ Universitat Politècnica de Catalunya (Spain) \\ oriol.lordan@upc.edu
}

\section{Abstract:}

Purpose: The network strategies used by airline carriers have been a recurring subject in air transport research. The aim of this paper is to investigate the relationship between the different operational characteristics of the airline and its route network configuration.

Design/methodology/approach: The two main airline carrier typologies - Full-Service and Low-Cost carriers - are analysed using empirical models developed on complex network research relating them to the business model of the airlines.

Findings and Originality/value: Just in Europe, one can differentiate between Full-Service and Low-Cost Carriers by complex network analyses. In this process, it has been also found that new concept Low-Cost Carriers, such as Vueling, have network properties closer to Full-Service Carriers.

Research limitations/implications: This paper has a limited sample, as includes 26 airline case studies from Europe, United States and Asia.

Practical implications: The analysis carried out in this research can help to the assessment of the evolution of the strategies of airline carriers, and has also operational implications, since the configuration of an airline route network can determine its resilience to attacks and errors. 
Social implications: A better understanding of the properties of airline route networks can benefit airlines, passengers and another stakeholders of the air transport industry.

Originality/value: Current research on air transport networks has only considered the global or regional level, but few studies have addressed the study of airline transport networks, and its relationship with their business model.

Keywords: complex networks, airline strategy, Full-Service Carriers, Low-Cost Carriers

\section{Introduction}

The commercial passenger air travel industry has changed dramatically in recent decades. It has evolved from a market controlled by state-owned airlines with little competition, to a more deregulated scenario, where a multitude of airlines compete intensely with different business models. Today's air transport is a demanding and competitive business, where margins are tight and is usual that some airlines record losses of millions (Cento, 2008).

Extant literature of airline strategy identifies three business models for airlines, clearly differentiated by its operational characteristics and services offered to passengers (Cento, 2008; Lordan, Sallan \& Simo, 2014). These are the models "Charter" for chartered flights, and the "Low-Cost Carrier" (LCC) and the "Full-Service Carriers" (FSC) for scheduled flights. Business models for scheduled flights have very different characteristics in terms of airline cost structure, services offered to customers, type of airports and route network configuration.

Configuration network of routes is a key element of an airline's strategy as it determines the structure of operating costs and may condition the strategy of competitors (Aguirregabiria \& Ho, 2010). The most widespread network configurations in the passenger air travel industry are the "point-to-point" (PP), the "hub-and-spoke" (HS) and the "multi-hub-and-spoke" (MHS). FSCs are traditionally considered to operate with a HS or MHS route network configuration (Wojahn, 2001) and that LCC routes are supposed to define a PP network. Nevertheless, to our notice no empirical examination of the network configuration of airline routes has been carried.

The aim of this research is to carry an analysis of the route configuration of a selected sample of airline carriers, known to be FSCs or LCCs, and operating in different regions. This analysis can be of interest to deepen the knowledge of the logic of airline business models. It can be also of interest for operational issues, since network configuration is a key feature in the resilience of airlines facing unintended network failures and intended attacks (Lordan et al., 2014; Lordan, Sallan, Simo \& Gonzalez-Prieto, 2014; Lordan, Sallan, Simo \& Gonzalez-Prieto, In press). 


\section{Airline network configurations}

In the hub-and-spoke (HS) network configuration all destinations are linked to a main airport called hub. A hub can be defined as an "airport where a large percentage of flights operated by an airline as part of a radio network" (Button \& Stough, 2000). The main advantages of this configuration are that it makes a great deal of origins and destinations relatively well interconnected with a low number of routes, and the possibility to achieve economies of scale and economies of density. It also offers the opportunity to apply economies of scope by centralizing in the hub the maintenance services and staff dedicated to aircraft operation. On the other hand, the fact that an airline has a particular airport as hub may deter other airlines from operating in this airport (Aguirregabiria \& Ho, 2010).

The HS network configuration has some drawbacks that might cause a significant increase in unit costs. First, operations in hubs can have a strong temporal density (i.e., the number of flights per unit of time is high), thus appearing traffic peaks. These peaks may generate traffic congestion in the hub, resulting in delays and increased turnover times of aircraft, raising airline unit costs and lowering service quality. Another drawback of the HS configuration is that connections between non-hub cities must pass through the hub, thus raising the travel time and the costs of fuel, crew and aircraft use. For that reason, it is not common that airlines adopt a "pure" HS network configuration, and they frequently schedule high demand routes not passing through the hub.

In the point-to-point (PP) configuration airports are connected by direct routes, rather than going through a central hub (Yan, Fu \& Oum, 2008). Usually, airlines do not implement a "pure" PP configuration: a fair proportion of their routes are planned from a set of base airports (Cento, 2008). Base airports are usually well connected to major cities, in which airlines centralize services such as aircraft maintenance and assistance to passengers, offering similar operational advantages as the hubs do in the HS configuration. However, since no connection services are offered, the temporal density of operations can be lower in bases than in hubs. As a consequence, airlines adopting a PP configuration have a lower probability of delays, lower peaks of needs of personnel and a lower turnover of aircrafts. The gains of efficiency obtained, together with the fact that secondary airports usually charge lower fees, makes those airlines adopting this network configuration more efficient.

The main disadvantage of the PP network configuration is the need to implement a much larger number of routes than the HS configuration to link a similar number of destinations. That's why airlines with PP network configuration usually operate only on routes where demand is high enough to have a high load factor on aircrafts. Another drawback of this network configuration is that long-haul routes are out of reach for airlines with PP networks, and are operated on hub airports of airlines adopting a HS or MHS configuration. 
With all these elements described, it seems logical that the PP network configuration is adopted by LCCs, because it allows a substantial cost reduction, but does not offer the possibility of providing some added value services offered in the HS configuration.

The multi-hub-and-spoke (MHS) network configuration is similar to the HS, but with several hubs instead of one. There are several factors leading to the adoption of a MHS configuration. It can be the result of the implementation of strategies of scale (Goedeking, 2010). This strategy can be adopted in locally fragmented markets, where it can be effective to maintain a hub for each region, and establishing strong and frequent connections between local hubs. This is the case of the US market, the first market where this configuration has been developed. A MHS can also appear in HS airlines when no additional routes can be added to the hub due to airport capacity limitations. Finally, a MHS configuration can also appear as a result of a merger and acquisition process. When the routes of several HS airlines are integrated in a single airline, a MHS configuration can appear.

The use of hubs allows the adopters of the MHS network to benefit from the same economies of scale, density and scope of the HS. The use of several hubs allows a more flexible consolidation of traffic in hubs through connecting flights, obtaining an adequate load factor for large aircrafts and efficient flight frequencies (Wojahn, 2002). On the other hand, the use of multiple hubs prevents the appearance of constraints due to insufficient airport capacity. The disadvantages of the MHS are similar to the HS, although there are some problems associated with the adoption of multiple hubs. The number of flights needed to connect two airports in a MHS can be larger than in the HS, since not all airports are connected to all hubs. There are also issues concerning the need to schedule a high rate of flights between multiple hubs.

The MHS configuration seems to be oriented to FSCs, due to its similarity with HS. In fact, this configuration is almost mandatory to airlines resulting from the merge of HS airlines, and to HS airlines with problems of capacity in their hubs. LCCs can also benefit from this structure when adopting a multi-base configuration. Table 1 illustrates the correspondence between airline business models and route network configurations.

\begin{tabular}{|l|l|}
\hline Business model & Network configuration \\
\hline Full-Service Carrier (FSC) & Hub-and-spoke (HS), multi-hub-and-spoke (MHS) \\
\hline Low-Cost Carriers (LCC) & Point-to-point (PP) \\
\hline
\end{tabular}

Table 1. Relationship between business models and network configurations 


\section{Study of centrality in airline networks}

The airline routes transport network is represented as an undirected, unweighted graph, whose nodes are the $n$ airports where the airline operates. The adjacency matrix $A$ of the graph has values $a_{i j}=1$ when the airline has at least one route connecting airports $i$ and $j$, and zero otherwise.

A measure of the importance of a node in a graph is the degree centrality for a node $C_{D}(i)$, defined as the number of edges linking the node $i$ :

$$
C_{D}(i)=\sum_{j=i}^{n} a_{i j}
$$

To determine to what extent an airline route network has a HS configuration, it can be of interest to assess the centralization of its graph. A graph is centralized when there is a single point more central than the rest. The graph of maximum centralization is a star, where $(n-1)$ nodes are all connected only to a central node, which represents a pure HS configuration. Freeman (1979) defined a normalized measure of graph centralization $C_{D}$, which equals 1 for a star and 0 for a non-centralized graph:

$$
C_{D}=\frac{\sum_{i}^{n}\left(C_{D}\left(i^{*}\right)-C_{D}(i)\right)}{(n-1)(n-2)}
$$

where $i^{*}$ is the node with the highest degree centrality.

Another measure of centrality of interest is betweenness centrality. The betweenness centrality assesses the number of times a node acts as a bridge along the shortest path between two other nodes. The expression of the normalized betweenness centrality for a node of an undirected graph is:

$$
C_{B}(i)=\sum_{j \neq k} \frac{g_{j k}(i)}{g_{j k}}
$$

where $g_{j k}$ is the number of geodesics linking $j$ and $k$, and $g_{j k}(i)$ equals the number of geodesics linking $j$ and $k$ containing $i$.

It is also possible to define a measure of graph centralization based on betweenness centrality which compares the graph with a star graph, the one where it takes its maximum value, in a similar way as the graph centralization based on degree (Freeman, 1979):

$$
C_{B}=\frac{\sum_{i}^{n}\left(C_{B}\left(i^{*}\right)-C_{B}(i)\right)}{n^{3}-4 n^{2}+5 n-2}
$$

where now $i^{*}$ is the node with the highest betweenness centrality. 
The centralization measures based on degree and betweenness should allow us to assess the configuration of airline networks. The highest values of centralization will correspond to airline networks organized in a HS or MHS configuration, while PP networks will exhibit lower values of centralization.

A way of assessing the presence of multiple hubs is through the analysis of the degree distribution of the graph, that is, the distribution of the probability $P(k)$ that a node has exactly $k$ links. Most airline networks are scale free, which means that the degree distribution approximates a power law $P(k) \sim k^{r}$. The degree exponent determines the role of hubs in the system. The smaller the value of $y$ the more important the role of hubs in the network.

When $y>3$, hubs are not relevant. If $2>y>3$ there is a hierarchy of hubs, with the most connected hub in contact with a small fraction of the nodes. For $y=2$ or smaller, a hub-andspoke network emerges, where the most relevant hub is in contact with a large fraction of the nodes (Barabasi \& Oltvai, 2004). Networks following a HS configuration will have values of $y$ close to 2, while PP networks will have values of $y$ closer to 3. The $y$ of MHS networks should lie somewhere in between the other two configurations. Table 2 summarizes the expected values of degree and betweenness centralization, and of the $\gamma$ parameter, for the three network configurations.

\begin{tabular}{|l|l|l|}
\hline Network configuration & Centralization & $\boldsymbol{Y}$ parameter \\
\hline Hub-and-spoke (HS) & High & Close to 2 \\
\hline Multi-hub-and-spoke & Moderate & Between 2 and 3 \\
\hline Point-to-point (PP) & Low & Close to 3 \\
\hline
\end{tabular}

Table 2. Relationship between network configurations and network parameters

\section{Results}

To assess the correspondence between business model and route network configuration, the route configuration of a selected sample of airline carriers, known to be FSCs or LCCs, and operating in different regions is analysed. 26 airlines operating in three regions were selected: Europe, United States and Asia. The list of airlines is reported in Table 3. 


\begin{tabular}{|l|l|l|}
\hline Region & FSC & LCC \\
\hline Europe & $\begin{array}{l}\text { Lufthansa (LH) } \\
\text { British Airways (BA) } \\
\text { Air France (AF) } \\
\text { Iberia (IB) }\end{array}$ & $\begin{array}{l}\text { Ryanair (FR) } \\
\text { easyjet (U2) } \\
\text { Vueling (VY) } \\
\text { Germanwings (4U) } \\
\text { Norwegian (DY) } \\
\text { Wizz Air (W6) }\end{array}$ \\
\hline United States & $\begin{array}{l}\text { American Airlines (AA) } \\
\text { Delta (DL) } \\
\text { United Airlines (UA) } \\
\text { US Airways (US) }\end{array}$ & $\begin{array}{l}\text { Southwest Airlines (WN) } \\
\text { AirTran Airways (FL) } \\
\text { Spirit Airlines (NK) } \\
\text { JetBlue Airlines (B6) }\end{array}$ \\
\hline Asia & $\begin{array}{l}\text { Cathay Pacific (CX) } \\
\text { Asiana Airlines (OZ) } \\
\text { Air China (CA) } \\
\text { Singapore Airlines (SQ) }\end{array}$ & $\begin{array}{l}\text { Air Asia (AK) } \\
\text { Lion Air (JT) } \\
\text { Cebu Pacific (5J) } \\
\text { JetStar Asia Airways (3K) }\end{array}$ \\
\hline
\end{tabular}

Table 3. Airlines included in the study

The route networks for the selected airlines were created from the routes of each airline scheduled from January 2010 to December 2011. For each airline, it is constructed the graph of the route network with data extracted in June 2010 from their route maps available on their online websites; with nodes representing airports and edges the routes scheduled between them. Then, centralization measures based on degree and betweenness, and the $y$ coefficient of the degree distributions had been computed for each airline. To obtain the $Y$, it is followed the procedure reported by Newman (2005), based on Zipf's law. The betweenness-based measure of centralization has been computed using node betweenness centralities computed with a cut-off value of three, since is unlikely that a passenger could schedule a flight with more than three connections.

Figure 1 includes the plots of betweenness centralization versus $Y$ for each of the three regions. In all cases there is a high correlation between degree and betweenness centralization, so this plots show all relevant information.

European airlines show a variety of network configurations: Lufthansa, Iberia and Air France have a HS route network, with low values of $y$ and high betweenness centralization. Wizz Air, Ryanair and easyJet have a PP network configuration, following the LCC business model. Interestingly, Vueling, Germanwings and Norwegian lie in between of the two models: all of them have moderate values of $y$ and values of betweenness centralization lower than the airlines with an HS configuration, thus revealing an MHS network configuration. The case of British Airways may show an evolution of an HS configuration, driven by the saturation of the London Heathrow hub. An examination of its betweenness centrality distribution shows that BA is driving part of its operations to the London Gatwick airport. In fact, the real use of London Gatwick by BA is mainly for point-to-point operations. The other airlines are LCCs with several bases covering a variety of point-to-point connections, although it must be noted that some of these airlines (e.g., Vueling) codeshare with FSCs, and offer connecting flights. A distinctive feature of European LCCs is that they plan their routes differently in the winter and the 
summer season. For instance, Ryanair increases its routes from London Stansted in winter, and increases its routes from Alicante in summer.
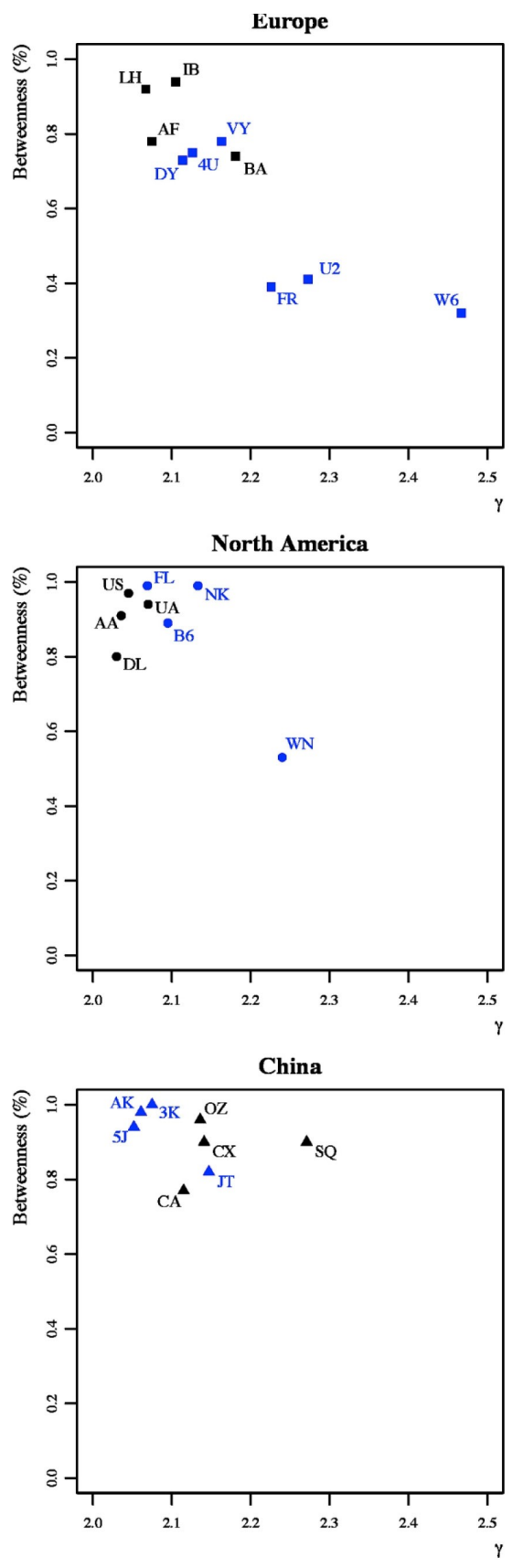

Figure 1. Betweenness centralization and $y$ for selected airlines

Among US airlines, Southwest Airlines shows a PP network, with a moderately high value of $y$ and a low betweenness centrality. All other airlines, being FSCs or LCCs, show a highly centralized network configuration. American Airlines, United Airlines and US Airways have low values of $y$ and high values of betweenness centralization, while Delta has a lower betweenness centralization, which may show that this airline has a high number of point-topoint routes over a HS configuration. The rest of LCCs (AirTran Airways, Spirit Airways and Jet 
Blue Airlines) have a route network centralized around a base, although the moderate value of the $y$ of Spirit Airlines suggests that this airline operates around several bases. Although the population distribution of the US may drive FSCs to adopt a MHS configuration, results show that FSCs tend to organize in a HS basis, complementing its route portfolio with alliance partners.

All Asian airlines have high values of betweenness centrality, showing that they tend to centralize its operations around a set of hubs or bases. Interestingly, LCCs have the lowest values of gamma, while FSCs have higher values. This fact suggests that Asian LCCs plan its route portfolio around a base, which is the origin and destination of most of its routes. Their route network is quite different from the European LCCS, which operate in several bases. It must be noted that Asian LCCs network structure may be limited by the bilateral restrictions; whereas European LCCs network structure may be affected by capacity shortage (consequently) at major airports, etc. Chinese carriers are still subject to entry regulation in domestic markets. Single versus multiple hub network are affected by the size of the network as well as regulatory constraints - domestic or international.

An examination of the degree distribution shows that the information provided by the $y$ parameter should be considered sceptically, though. For instance, consider Lufthansa $(y=2.067)$, and Singapore Airlines $(y=2.271)$. An examination of the airports with highest betweenness centrality in the Lufthansa route network shows that this airline operates in two hubs (Frankfurt and Munich). On the other hand, a similar examination of the most central airport of Singapore Airlines route network shows that this airline has a single hub in the Singapore airport. Another interesting example is American Airlines. Although it has high betweenness centralization and a low gamma $(y=2.036)$, this airline really operates with a MHS model, with up to three hubs (Dallas, Miami and Chicago).

\section{Conclusions}

This study shows that the analysis of airlines route networks contributes to deepen our knowledge of how airlines following different business models operate. It has been shown that the route networks of FSCs and LCCs are scale-free, with a degree distribution following a power law. This result is contrary to some a priori assumptions made by previous research, which suggested that the route network of LCCs should behave as a random network (Cento, 2008). The difference between the two business models lies in the role played by airports with high degree and betweenness centrality. While in FSCs these airports act as hubs centralizing connected flights, in LCCs these airports act as bases where these carriers centralize their maintenance operations. 
The results of the study show also differences among regions, especially in airlines following a LCC business model. The most mature model has resulted to be the European market. In this region it can be found airlines acting as FSCs, with a HS or a MHS configuration. The later configuration can appear when the main hub is saturated. This is the case of British Airways, a carrier that has created an alternate hub in London Gatwick to centralize its point-to-point connections probably for capacity problems in London Heathrow. Among the European LCCs examples of a "pure" PP route network can be found, with significant differences between the summer and winter route portfolio. Among the European LCC a set of airlines is appearing (Vueling, Germanwings and Norwegian) that are developing a hybrid business model that lies between FSC and LCC (Lohmann \& Koo, 2013). The business model of these airlines can be characterized as CORIC (Customer Oriented at Right Cost), since they offer a passenger experience similar to LCC, but with some traits of FSC. Some of these airlines (e.g., Vueling), although they are known to be "low-cost", are beginning to offer connecting flights and have codeshare agreements with FSCs. American and Asian carriers tend to have a more centralized network configuration than their European counterparts, with the exception of Southwest, who follows a PP configuration, with a low degree and betweenness centralization.

With respect to network route measures, the most useful has resulted to be betweenness centralization, which helps to tell airlines following a HS or MHS business model from the ones following a PP business model. The use of $y$ as a measure for detection of a multi-hub configuration has not been so useful, since some airlines adopting MHS model have low values of that parameter. The examination of the airports with highest values of centralities in the route network allows detecting more precisely the main airports of the route network, being their hubs or bases.

Further studies should consider analysing a wider variety of airlines from different regions, paying attention not only to centralization measures for the whole network, but also to degree and betweenness distributions. Longitudinal analyses could also shed light in order to capture the effects of frequency, which is an important aspect of network configuration of FSCs and LCCs. These studies can also benefit from methodologies from the business models literature, incorporating to the route network analysis some other elements of airline strategy, such as customer service and participation in alliances. 


\section{References}

Aguirregabiria, V., \& Ho, C.Y. (2010). A dynamic game of airline network competition: Hub-and-spoke networks and entry deterrence. International Journal of Industrial Organization, 28(4), 377-382. http://dx.doi.org/10.1016/j.ijindorg.2010.03.003

Barabasi, A.L., \& Oltvai, Z.N. (2004). Network biology: understanding the cell's functional organization. Nature Reviews Genetics, 5(2), 101-113. http://dx.doi.org/10.1038/nrg1272

Button, K.J., \& Stough, R. (Eds.). (2000). Air transport networks: Theory and policy implications. Edward Elgar Publishing.

Cento, A. (2008). The airline industry. Springer.

Freeman, L.C. (1979). Centrality in social networks conceptual clarification. Social networks, 1(3), 215-239. http://dx.doi.org/10.1016/0378-8733(78)90021-7

Goedeking, P. (2010). Networks in aviation. Strategies and Structures. Berlin. http://dx.doi.org/10.1007/978-3-642-13764-8

Lordan, O., Sallan, J.M., \& Simo, P. (2014). Study of the topology and robustness of airline route networks from the complex network approach: a survey and research agenda. Journal of Transport Geography, 37, 112-120. http://dx.doi.org/10.1016/j.jtrangeo.2014.04.015

Lordan, O., Sallan, J.M., Simo, P., \& Gonzalez-Prieto, D. (2014). Robustness of the air transport network. Transportation Research Part E: Logistics and Transportation Review, 68, 155-163. http://dx.doi.org/10.1016/j.tre.2014.05.011

Lordan, O., Sallan, J.M., Simo, P., \& Gonzalez-Prieto, D. (In press). Robustness of Airline Alliance Route Networks. Communications in Nonlinear Science and Numerical Simulation. http://dx.doi.org/10.1016/j.cnsns.2014.07.019

Lohmann, G., \& Koo, T.T. (2013). The airline business model spectrum. Journal of Air Transport Management, 31, 7-9. http://dx.doi.org/10.1016/j.jairtraman.2012.10.005

Newman, M.E. (2005). Power laws, Pareto distributions and Zipf's law. Contemporary physics, 46(5), 323-351. http://dx.doi.org/10.1080/00107510500052444

Wojahn, O.W. (2001). Airline network structure and the gravity model. Transportation Research Part E: Logistics and Transportation Review, 37(4), 267-279. http://dx.doi.org/10.1016/S1366-5545(00)00026-0

Wojahn, O.W. (2002). The impact of passengers' preferences regarding time and service quality on airline network structure. Journal of Transport Economics and Policy, 139-162. 
Yan, J., Fu, X., \& Oum, T. (2008). Exploring network effects of point-to-point networks: An investigation of the spatial entry patterns of Southwest Airlines (No. 2008-21).

Journal of Industrial Engineering and Management, 2014 (www.jiem.org)

\section{(a)}

Article's contents are provided on a Attribution-Non Commercial 3.0 Creative commons license. Readers are allowed to copy, distribute and communicate article's contents, provided the author's and Journal of Industrial Engineering and Management's names are included. It must not be used for commercial purposes. To see the complete license contents, please visit http://creativecommons.org/licenses/by-nc/3.0/. 